\title{
Cáncer del Cervix y sus Factores de Riesgo Estudio de Casos y Controles
}

\author{
Licenciada Luz Zuloaga Posada*
}

\author{
Licenciada Cecilia Soto Vélez*
}

Colaboradores:

Estudiantes Epidemiología

Facultad de Enfermería

Universidad de Antioquia

1980

\section{INTRODUCCION}

En la actualidad se postulan como teorías causales del cáncer de cervix los siguientes factores: viral, genético-inmunológico, hormonal, la irritación o infección crónica y sustancias carcinogenéticas; además, se habla de infinidad de variables relacionadas con la actividad sexual como posibles indicadores de los factores de riesgo en este tipo de cáncer. Estas teorías están respaldadas por infinidad de estudios consistentes que demuestran asociaciones estadísticas y que están en concordancia con los conocimientos actuales, $(1,2,3,4,5,6,7)$.

En cuanto al factor viral como agente causal, Nahmias (1) informa que el 90 por ciento de los casos tenían virus HSV-2, siendo mayor la frecuencia de infección en las prostitutas y con mayor número de compañeros sexuales. David B. Thomas (8) informa que la frecuencia de títulos de anticuerpos contra el herpes virus II es 2.28 veces mayor y en for-

* Profesoras de Epidemiología, Facultad de Enfermería, Universidad de Antioquia. ma significante para mujeres con cáncer de cervix al compararla con la frecuencia de los controles. Tobin (9) al provocar infección en el laboratorio en células de cuello de útero humano y de animales, ambos tejidos desarrollaron hipertrofia en citoplasma y en el núcleo celular. Los investigadores explican que el virus penetra la célula y en el protoplasma de ésta, comienza a multiplicarse, ocasiona la muerte de la célula invadida atacando nuevas células produciendo así enfermedad viral herpética, hasta que entran en acción las defensas inmunitarias impidiendo la difusión posterior a otra célula ; pero el virus puede penetrar a la célula y no quedarse en el protoplasma penetrando al núcleo de ésta, mezclándose con el material genético celular, modificándolo, manteniendo su apariencia normal por cierto tiempo, para luego empezar a reproducirse de manera incontrolada.

Dentro de la actividad biológica, los organismos vivientes se encuentran en un permanente intercambio celular $y$ así producen millones de células nuevas diariamente para remplazar a otras que deshecha. Investigadores han encontrado en estas células deshechadas algunas mutaciones compatibles con malignidad, argumentándose que el organismo por

\footnotetext{
Trabajo presentado en el IV Congreso Colombiano de Cancerología, 1-5 de septiembre de 1981 Bogotá, D.E. - Colombia.
} 
propiedad genética y a través del sistema inmunológico, se encuentra vigilante de estas transformaciones celulares para eliminarlas $y$ al fallar este sistema protector, las células aberrantes quedan sin control multiplicándose rápidamente y determinando la neoplasia (10). Esta teoría se ve apoyada por el aumento de la frecuencia del cáncer en los estados caracterizados por bajas defensas inmunológicas como son la vejez, el embarazo, y el uso prolongado de inmunosupresores. En la actualidad algunos investigadores creen que la baja frecuencia del cáncer de Cervix en Israel se debe a patrón genético especial (11).

Peritz, (12) en el Norte de California, en un estudio prospectivo encontró asociación positiva entre tiempo de consumo de anticonceptivos orales y carcinoma de cervix uterino después de controlar ocho variables de confusión. En el mismo sentido Saulny (13) informó en Venezuela una frecuencia de 24.15 por 10.000 mujeres en una cohorte de 14.891 mujeres año-uso de anticonceptivos inyectables. Mattingly (14) informa asociación positiva entre cáncer de cervix $y$ Dietiletilbestrol (DES) con una $\mathrm{P}=0.05$ y un riesgo relativo de cinco. Herbest (15) en USA encontró que en 384 mujeres con Dx. de cáncer de cervix y vagina, el $61 \%$ ten ían antecedentes de DES durante la vida intrauterina, encontrándose mayor riesgo de desarrollar cáncer en las mujeres cuyas madres recibieron DES en las primeras semanas de gestación.

En varios países de Europa, Asia y Norte América se han hecho infinidad de estudios analíticos para conocer los factores de riesgo del cáncer de cervix uterino; de los realizados en Colombia se han publicado datos referentes a los factores de riesgo de la enfermedad, en Cali, realizados por Guzmán y Guerrero (2), Muñoz y otros (16); Ney Guzmán (17) informó que la frecuencia de este tipo de cáncer en Cali en 1963 fue de 66.4 por $100.000 \mathrm{~h}$; la tasa promedio de mortalidad por cáncer del cervix en el Departamento de Antioquia para el período de 1966-1976 fue de $5.57 \times$ 100.000 h. (18). Esta frecuencia tan alta de la enfermedad justifica la realización de estudios analíticos que aporten al conocimiento de la Historia Social del cáncer de cervix en todo el pais y en cada una de sus regiones. Por tal razón los objetivos del estudio tienden a describir las características de la población con cáncer de cervix que demandan atención en el Departamento de Oncología del Hospital San Vicente de Paúl de la ciudad de Medell ín y compararlo con las características de las mujeres que no tienen cáncer y que demandan servicio de citología en esta misma institución. El estudio también pretendió describir la etapa de evolución del cáncer de cervix en la cual se hace el diagnóstico en la población de estudio.

\section{METODOLOGIA}

La metodología empleada fue de Casos y Controles. La realización de estudios poblacionales que permitan medir la incidencia de cáncer de cervix en la ciudad de Medellín presenta serias dificultades debido principalmente a varios factores: No toda la población tiene acceso a los servicios de atención médica, la información existente en las distintas instituciones de salud no fue posible utilizarla porque las directivas de alguna de ellas se negaron rotundamente a suministrar la información. La población de referencia es aquella que solicita servicios de atención ginecológica al laboratorio de anatomía patológica y al servicio de Oncología del Hospital San Vicente de Paúl de la ciudad de Medellín. Al servicio de Oncología de esta institución son remitidas pacientes diagnosticadas con cáncer de cervix en el Hospital San Vicente de Paúl, Pablo Tobón Uribe, Instituto Seguro Social, Profamilia, Ca- 
precan, Médicos particulares y Hospital General de Medellín.

El grupo de casos consistió en $81 \mathrm{mu}$ jeres con diagnóstico de cáncer de cervix comprobado por anatomía patológica realizado durante 1979 , enero y febrero de 1980, inscritos en el registro de control de cáncer ginecológico realizado por el grupo de Oncología ginecológica del Hospital San Vicente de Paúl de la ciudad de Medellín. Las 81 mujeres aparecían en dicho registro como residentes en Medellín.

Se seleccionaron 162 controles, del total de resultados de citologías de mujeres residentes en Medell ín con informe negativo, grado I ó grado II, sin atipias procesadas en el laboratorio de Anatomía Patológica del Hospital San Vicente de Paúl, dentro del mismo período del diagnostico de los casos.

Debido a que la población de controles era más joven que la población de casos, se equiparó por edad con un rango de más o menos cinco años.

La información para casos y controles se tomó a través de entrevista personal realizada por enfermeras estudiantes de Epidemiología de la Facultad de Enfermería de la Universidad de Antioquia. La entrevista se realizó en el lugar de residencia y controlando la duración de la misma; al formulario se adicionaron preguntas no relacionadas con el cáncer de cervix con el fin de controlar la calidad de la información. Si la persona había muerto al momento de la entrevista, se interrogó al familiar más cercano y si había cambiado de domicilio, la encuesta se envió por correo; también se utilizó como fuente de información adicional la historia clínica de cada caso.

Se estudiaron como factores de riesgo los siguientes: nivel socioeconómico medido a través de la ocupación y el ingreso mensual percápita, edad de la menarca, del primer embarazo, de iniciación de actividad sexual, número de embarazos y abortos, consumo de hormonas y esteroides, uso de anticonceptivos, antecedencia de infecciones genitales, frecuencia de relaciones sexuales y número de compañeros sexuales.

El análisis estad ístico se hizo en aqueIlas variables en las cuales la tasa de respuesta fue similar para casos y controles, usando como estadígrafos de contraste diferencia de proporciones.

$$
\left(Z=\frac{P_{1}-P_{2}}{\sqrt{\frac{P_{1} q_{1}}{n_{1}}+\frac{P_{2} q_{2}}{n_{2}}}}\right)
$$

Diferencia de Promedio $=\frac{\bar{x}_{1}-\bar{x}_{2}}{\sqrt{\frac{1}{2} n_{1}+\frac{2}{n_{2}}}}$

Para las variables en las cuales el índice de respuesta fue menor en los casos que en los controles se hizo análisis de pareo con el estad ígrafo Chi-Cuadrado:

$$
x^{2}=\frac{(R-S)^{2}}{R+S}
$$

\section{RESULTADOS}

Como se puede observar en el gráfico No. 1 el $55.56 \%(32.10+23.46 \%)$ de los casos de cáncer de cervix atendidos en la Unidad de Oncología ginecológica del Hospital San Vicente de Paúl de Medellín, se encontraban en la quinta y sexta década de la vida.

Esto no indica propiamente el comportamiento de la enfermedad con relación a la edad, sino la edad en la cual se hace el diagnóstico en nuestro medio. Al analizar la edad de acuerdo con el esta- 
EDAD EN MUJERES CON CANCER DE CUELLO UTERINO

H.S.V.P. MEDELLIN L979-1.980

GRAFICO NN I

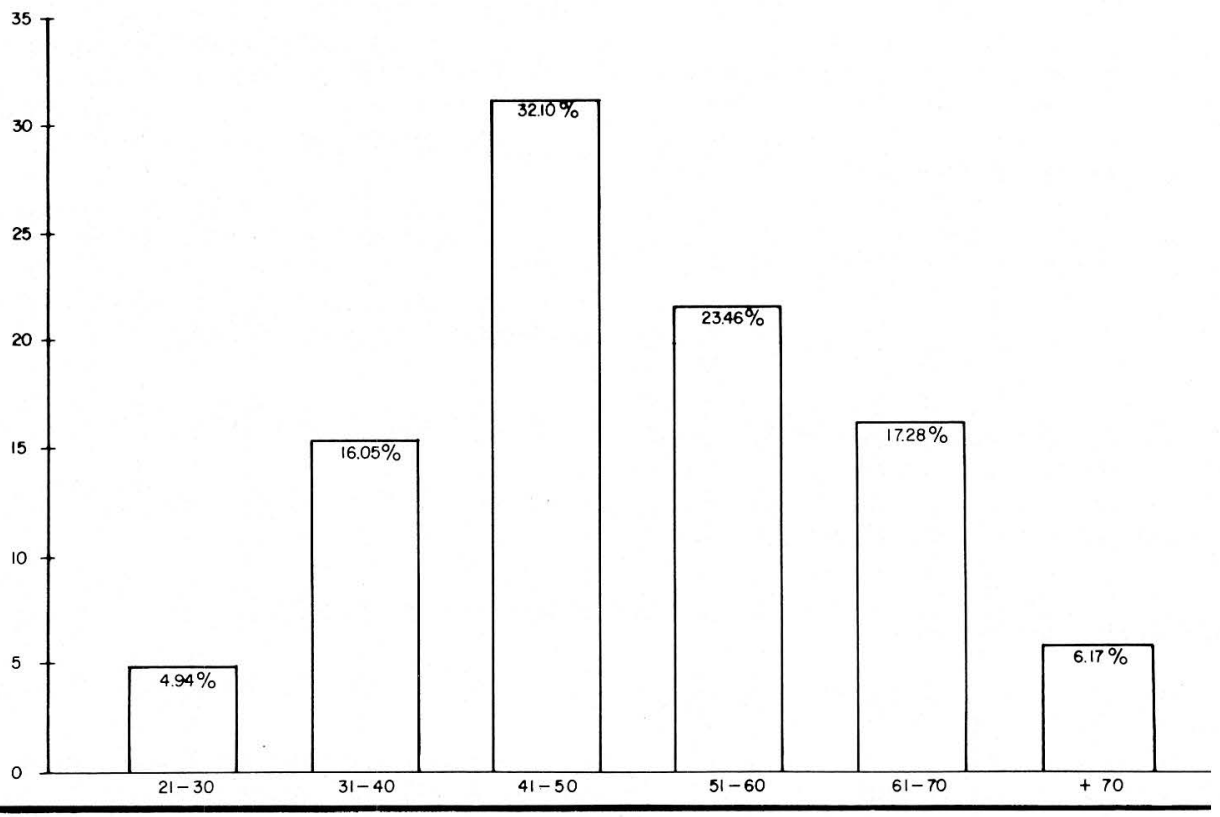

GRAFICO № 2

PORCENTAJE DE EDAD SEGUN DIAGNOSTICO DE CANCER DE CUELLO

H.S.V.P. MEDELLIN $1.979-1.980$

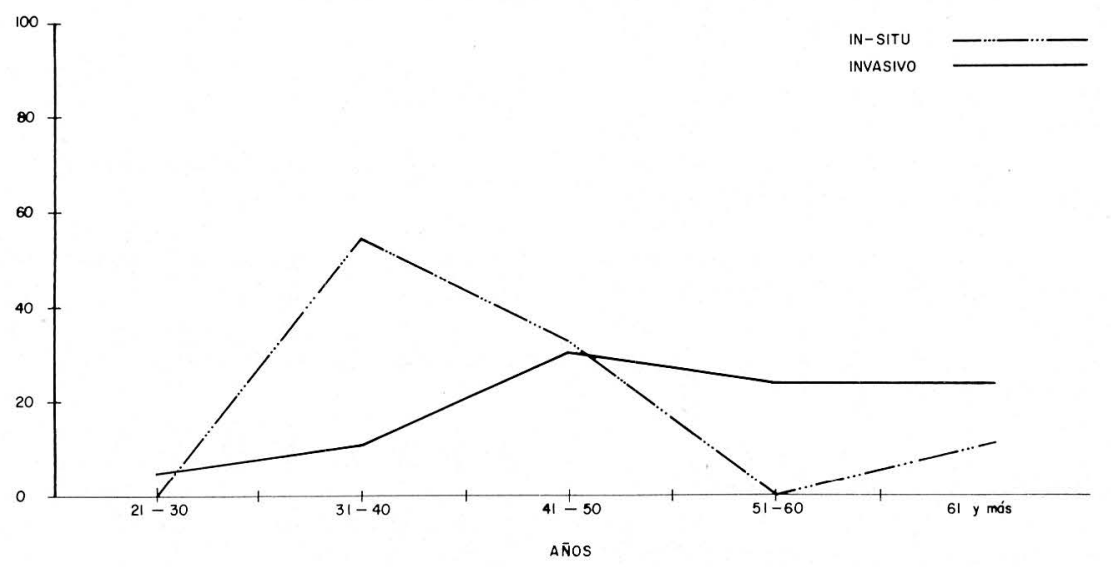


dío de evolución, el cáncer in-situ aparece en edades (una década) más tempranas que el cáncer invasor (gráfico No. 2).

\section{Características socio-económicas:}

Para medir la variable socio-económica en las mujeres con cáncer de cervix, se obtuvo un índice de respuesta del 50 por ciento aproximadamente para los siguientes indicadores: Ocupación del jefe de la familia, ingreso per cápita y escolaridad de la señora y su compañero. En cuanto a la ocupación se encontró que el 61.11 por ciento de los jefes de familia eran obreros y el $\mathbf{3 3 . 3}$ por ciento eran desempleados; el ingreso per cápita osciló en un rango entre $\$ 100.00$ y $\$ 4.000 .00$ mensuales presentando la siguiente distribución: 54.55 por ciento tuvieron ingresos de $\$ 100.00 \quad a$
$\$ 1.000 .00$; en el 36.36 por ciento el ingreso fue de $\$ 1.001 .00$ a $\$ 2.000 .00$ y el 9.09 por ciento ganaban de $\$ 2.001 .00$ a $\$ 4.000 .00$. Para medir la escolaridad se usaron los criterios de analfabeto, cuando no sabía leer ni escribir, alfabeto cuando sabía leer y escribir o había cursado algún grado de escuela primaria; con formación básica primaria, quien hubiese terminado todo el ciclo. Con relación a esta variable se encontró que el 21.05 por ciento eran analfabetos, 31.58 por ciento alfabetos, el 47.37 por ciento habían terminado la escuela primaria. En las mujeres el más alto grado de escolaridad fue el 5o. de bachillerato $y$ en los jefes de familia fue la preparación técnica. Con base en lo anterior podemos concluir que el nivel económico de los casos fue muy bajo; sin embargo, no puede considerarse como una carac-

GRAFICO $N^{\circ} 3$

CLASIFICACION ANATOMOPATOLOGICA DEL CANCER DE CUELLO

AL MOMENTO DEL DIAGNOSTICO

H.S.V.P. MEDELLIN I.979-1.980

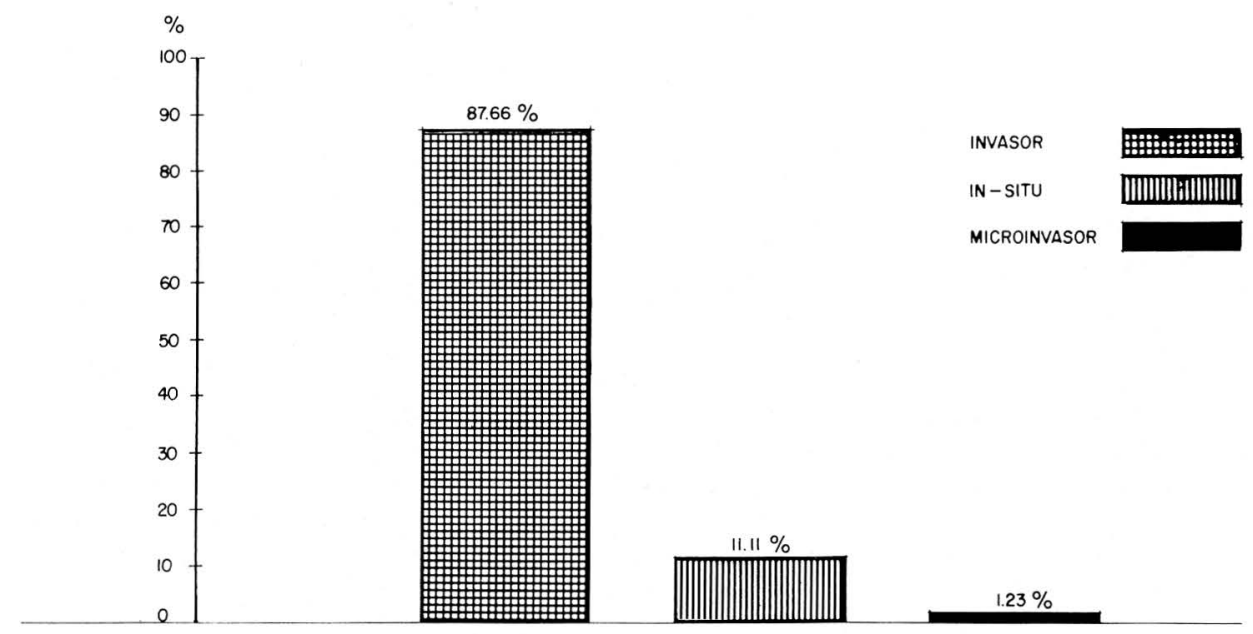


terística de la enfermedad sino de la población atendida por esta institución de salud.

Según el diagnóstico histo-patológico el $87.66 \%$ de los casos eran cáncer invasor lo que demuestra lo tardío del diagnóstico en nuestro medio (gráfico No. 3), Evaluadas las etapas del desarrollo del cáncer según diagnóstico clínico, se encontró que el $60.73 \%(59.21+1.52)$ de Ios casos estaban en grado IIIB y IV (gráfico No. 4).

\section{GRAFICO No4}

CLASIFICACION CLINICA DEL CANCER DE CUELLO

AL MOMENTO DEL DIAGNOSTICO

H.S.V.P. MEDELLIN $1.979-1.980$

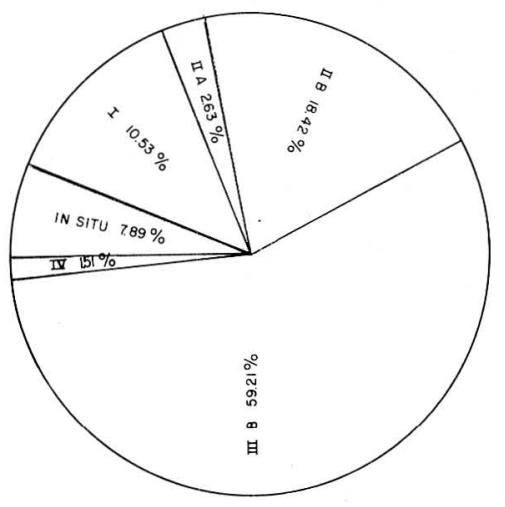

El índice de entrevista personal en los casos fue aproximadamente del 50 por ciento $\mathrm{y}$ en los controles del 100 por ciento; esta diferencia puede explicarse porque una gran proporción de casos (20.99 por ciento) ya había muerto al momento de la entrevista, razón por la cual tuvo que encuestarse a algún familiar cercano que estuviese presente, varios pacientes habían cambiado de sitio de residencia y aunque se les envió el formulario ninguno respondió. Este hecho hizo que casos y controles no fueran comparables con relación a algunas variables consideradas factores de riesgo para cáncer de cervix y que fueron detalladas en la metodología del estudio. Con el fin de recuperar información en los casos se acudió a consultar la historia clínica del paciente y la información procesada en la Universidad de Antioquia conducente a un estudio sobre cáncer de cervix que adelanta el grupo de Oncología ginecológica. La recuperación de la información a través de estas fuentes para las variables que a continuación se analizan fue de 100 por ciento, menos para el lugar de residencia y edad de la menarquia que fueron $91.3 \%$ y $75.2 \%$ respectivamente.

EI equiparamiento por edad fue efectivo resultando sus promedios iquales estad ísticamente $(Z=0.103, P=0.92)$.

En cuanto a los factores de riesgo denominados como comportamiento sexual se encontró una edad significativamente menor a la primera relación sexual y al primer embarazo en los casos que en los controles; la probabilidad de ser gran multípara también fue mayor significativamente en los casos que en los controles. Con relación al promedio de la edad de la menarquia no se encontró diferencia significativa aunque el valor de $\mathrm{P}$ para el estadígrafo de contraste estuvo cerca al límite de significancia estadística, (gráficos Nos. 5 y 6 y cuadro No. 1).

La procedencia de Zona Urbana fue mayor en los controles que en los casos, siendo significativa esta diferencia $(\mathrm{P}=0.000009)$.

Para los factores de riesgo en los cuales el porcentaje de no respuesta fue mucho mayor en los casos se hizo análisis de pareo. Las variables, número de compañeros y frecuencia de relaciones sexuales por semana, no se analizaron por la inconsistencia de la información. No se encontró asociación en ninguna de estas variables, (cuadro No. 2 ). 
COMPARACION DE CASOS Y CONTROLES

PARA FACTORES DE RIESGO DE CA DE CUELLO

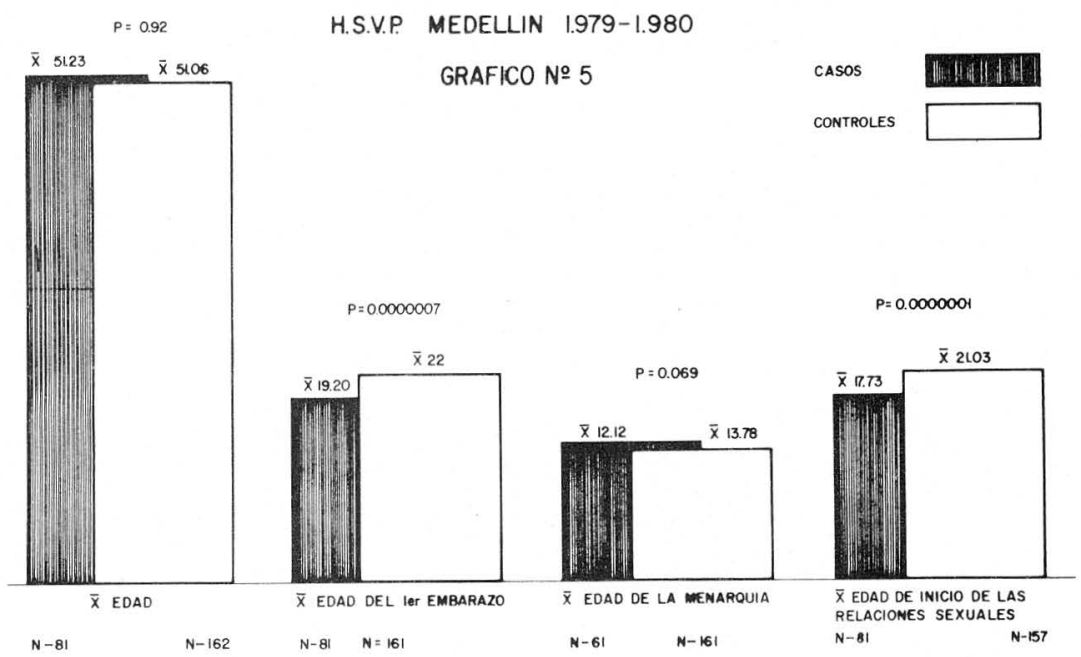

GRAFICO № 6

COMPARACION DE CASOS Y CONTROLES

PARA FACTORES DE RIESGO DE CA. DE CUELLO

H.S.V.P. MEDELLIN 1.979-1.980

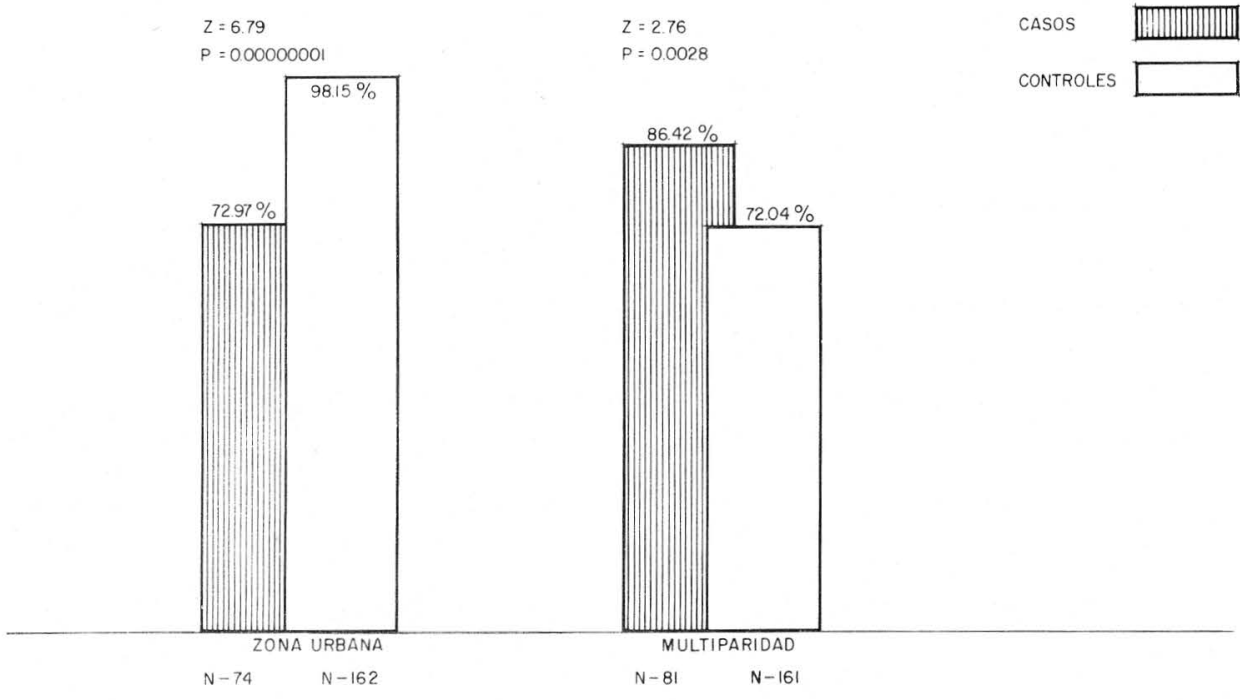




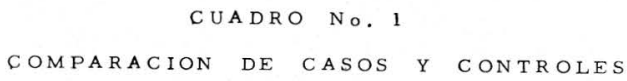

\begin{tabular}{|c|c|c|c|c|}
\hline CARACTERISTICAS & CASOS & CONTROLES & $z$ & $\mathrm{P}$ \\
\hline $\begin{array}{l}\text { Promedio edad } \\
\text { prime } r \text { e mba razo }\end{array}$ & $\begin{array}{l}19.20 \\
D_{3}: \pm 3.53 \\
n: 81\end{array}$ & $\begin{array}{l}22 \\
\text { Ds: } 5.37 \\
n: 161\end{array}$ & 4.82 & 0.0000007 \\
\hline $\begin{array}{l}\text { Promedio edad de } \\
\text { la menarquia. }\end{array}$ & $\begin{array}{l}14.12 \\
\text { D: }: \pm 1663 \\
n: 61\end{array}$ & $\begin{array}{l}13.78 \\
\text { Ds: } \frac{ \pm}{n} 1.58 \\
\text { n: } 161\end{array}$ & 1.48 & 0.069 \\
\hline $\begin{array}{l}\text { Promedio edad } \\
\text { inicio relaciones } \\
\text { sexuales. }\end{array}$ & $\begin{array}{l}17.73 \\
\text { Ds: } \pm 3.54 \\
\text { n: } 81\end{array}$ & $\begin{array}{l}21.03 \\
\text { Ds: } 5.66 \\
n: 157\end{array}$ & 5.10 & 0.0000001 \\
\hline $\begin{array}{l}\text { Probabilidad de } \\
\text { gran multíparas } \\
\text { (más de } 5 \text { emba- } \\
\text { razos). }\end{array}$ & $\begin{array}{l}86.42 \% \\
\text { n: } 81\end{array}$ & $\begin{array}{l}72.04 \% \\
n: 161\end{array}$ & 2.76 & 0.0028 \\
\hline$\%$ Zona Urbana & $\begin{array}{l}72.97 \% \\
\text { n: } 74\end{array}$ & $\begin{array}{l}98.15 \\
\mathrm{n}: 162\end{array}$ & 6.79 & 0.00000001 \\
\hline
\end{tabular}

ANALISIS DE PAREO DE FACTORES DE RIESGO GINECO-OBSTETRICOS

H. U.S.V.P. MEDE LLIN, $1979-1980$

FACTORES DE RIESGO

Mŕs de un aborto

Trastornos menstruales

Consumo de anorulatorios

Infecciones genitales

Antecedentes familia res de

cánce $\mathbf{r}$
NUMERO DE PA -

REJAS.

0.8

0.2 


\section{DISCUSION}

El promedio de edad al momento del diagnóstico de cáncer de cuello en la población atendida en el Servicio de Oncología ginecológica del Hospital San Vicente de Paúl de Medell ín (H.U.S.V.P.) fue de 51.23 con una desviación estándar de más o menos 12.41 años, siendo más frecuentes en la quinta y sexta década de la vida. La menor edad a la cual se hizo el diagnóstico fue de 28 años. Uribe, Duque (19) en Medell ín informa que el $55.56 \%$ de los pacientes tenían de 41 a 60 años, siendo el promedio de edad de 43.5 años para cáncer general; de 46.83 años para invasor y 37.5 años para cáncer in-situ. Botero Uribe, en Medellín (20) encontró que el $84.3 \%$ de los casos se diagnosticaron entre los 31 y 60 años. Baquero (21), también en Medellín, encontró que la mayoría de los casos de cáncer in-situ en el H.U.S.V.P., tenían entre 30 y 49 años. Ney Guzmán, en Cali (17) encontró el 52.8 por ciento de casos de cáncer de cervix con 35 y 49 años y 28.4 por ciento de los casos tenían entre 50 y 64 años. Henao, en Cali (22) encontró que el 87 por ciento de los casos de cáncer cervicouterino ten ían entre 45-54 años. Erazo (23), en Popayán encontró como edad más frecuente para casos de cáncer en general la de 45 y más años y para cáncer in-situ entre 25 a 29. En el Hospital San Juan de Dios en Bogotá, Fonnegra (24) encontró un promedio de edad de 41.2 años para casos de carcinoma infiltrante de cuello de útero. Rengifo (25) encontró una frecuencia de 45.7 por ciento de cáncer in-situ en mujeres de 30-39 años y de 41.2 por ciento de cáncer invasor en mujeres de 40-49 años. Barros (26), en Medellín, observó en mujeres con cáncer in-situ el $79.4 \%$ tenían de 30 a 49 años. En un trabajo cooperativo, presentado en el VI Congreso Nacional de Cirugía, en Medellín (27) informaron como edades más frecuentes para cáncer de cervix los 35 y 39 años, siguiendo en frecuencia las edades de 40 a 50 años. En Chile, Caorsi (28) encontró un promedio de edad de 48.9 años para carcinoma invasor y de 38.6 años para cáncer in-situ, edad menor que la reportada por este estudio, en el cual el promedio de edad para cáncer invasor fue de 52.12 con una desviación estándar de 12.38 y para cáncer in-situ, el promedio de edad fue de 43.55 años con una desviación estándar de 12.38. Alert (29) en Cuba, encontró como edad media en mujeres ccn cáncer cervicouterino, 51.1 años. Chisto-Pherson M. y otros, (30) informó como edad media para pacientes con cáncer de cuello de útero los 42 años; para cáncer in-situ 38.2 años; para carcinoma invasivo 51.8 años.

En el presente estudio, se observó que 88.81 por ciento de los pacientes que iniciaron tratamiento durante el período de estudio ya tenían un cáncer invasor, frecuencia similar $(87.2 \%$ ) observó Ney Guzmán (2) en Cali, al seleccionar los casos para un estudio sobre cáncer de cervix, Rengifo (25) en Ibagué, en 80 casos hospitalarios encontró el $\mathbf{4 2 . 5}$ por ciento de cáncer invasivo y el resto de cáncer cervical in-situ. Uribe (19) en Medellín, observó una proporción de $68.8 \%$ de cáncer invasor, en casos hospitalarios. Nealon (5) en un grupo de 75.480 mujeres incluidas en un seguimiento para detección temprana de cáncer de cervix en Lanisville, encontró para todas las edades una probabilidad de cáncer de cervix tipo invasor de 0.24 por ciento y 0.30 por ciento de cáncer in-situ. Cuando analizó las mujeres menores de 20 años no encontró ningún tipo de cáncer y en las mujeres de 21 a 29 años, encontró tasas de $1.4 \times 1.000$ para cáncer in-situ y solamente 0.03 por ciento de cáncer invasor. Ramírez, (31) en México, en casos hospitalarios encontró frecuencias de 32.1 para cáncer in-situ y 12.3 por ciento para carcinoma infiltrante. Fonnegra (24) de 58 casos revisados encontró que 93 por ciento (54 casos) eran 
carcinoma infiltrante. En Chile, Caorsi (28) reportó un 22.60 por ciento de carcinoma invasor en 5.813 biopsias, cervico-uterino. Marcos (32) en España, también reportó mayor frecuencia de cáncer invasor que cáncer in-situ en una relación de 3.67 casos de cáncer cervical invasor que uno de cáncer in-situ y Ruch (33) encontró frecuencias relativas de 52.63 por ciento y $\mathbf{4 0 . 2 4}$ por ciento para cáncer cervical in-situ e invasor respectivamente, considerando el último como invasión de 6 a $10 \mathrm{~mm}$.

La edad de la menarquia, analizada como elemento del comportamiento gineco-obstétrico, no parece contribuir al cáncer de cervix, aunque el promedio de edad de los casos (14.12) fue mayor que el promedio de edad de controles (13.78) no fue significativa estad ísticamente esta mayor edad de la menarquia de los casos. Aure (34) en Venezuela, encontró que la mayor incidencia de cáncer de cuello uterino fue en mujeres cuya menarquia ocurrió entre 13 y 14 años y Bedoya (35) en el Perú tampoco encontró relación entre cáncer de cuello y la edad de la menarquia.

En el estudio de casos y controles realizado en el Hospital San Vicente de Paúl se encontró que la edad promedio del primer embarazo en los casos era menor significativamente que el promedio de edad de los controles, 19.20 años y 22 años respectivamente, este hallazgo coincide con lo reportado por Ney Guzmán (2) en Cali en cuyo estudio encontró edades promedios para el primer parto de 19.6 años para los casos y 21.1 años para los controles, Barros (26) en MedeIlín, de una observación de 145 historias clínicas con diagnóstico de cáncer in-situ concluyó que el $29 \%$ había tenido su primer embarazo antes de los 17 años. Bedoya (35) en el Perú, Erazo (23) en Colombia y Nealon en U.S.A. (5) apoyan la teoría de la asociación entre edad temprana del primer hijo y cáncer de cervix.

En el mismo sentido, en el presente estudio, se encontró asociación entre edad temprana al iniciar la vida sexual y el cáncer cervical, para los casos la edad promedio fue de 17.73 años y para los controles 21.03 años con una probabilidad de error de 0.0000001 estando de acuerdo con otros autores en la experiencia Nacional e Internacional, (14, 2, 7, $8,34,35,26)$, Kodama (37) en un artículo sobre relaciones entre aspectos epidemiológicos y endocrinos del cáncer cervical, cita a Rotkin y Wibanks como defensores de esta misma teoría, Wibanks presenta a discusión la posibilidad de que un agente carcinogénico relacionado.con el coito puede tener acceso a células inmaduras del cuello uterino que son susceptibles durante la metaplasia escamosa activa de la adolescencia, sin embargo, en este mismo artículo, Kodama cita a Jordán quien no encontró asociación sobre edad temprana al primer coito y cáncer de cuello uterino.

Como en otras series de pacientes a nivel Nacional e Internacional $(2,21$, $24,26,27,31,34,35)$ la mayor multiparidad se ha encontrado asociada a cáncer de cuello uterino, en la serie de pacientes analizadas en este estudio se encontró en los casos una probabilidad de gran multiparidad ( 5 + embarazos) de 86.42 por ciento contra una probabilidad de gran multípara en los controles de 72.04 por ciento, la diferencia fue significativamente estadística.

Aunque la literatura revisada $(1,2,3$, $4,5,6,7,8,9,12,13,14,15,16$ ) revela consistentemente asociaciones entre cáncer cervicouterino y una serie de factores de riesgo como consumo de anticonceptivos orales y hormonas, mayor frecuencia de relaciones sexuales, antecedentes de infección genital, bajo nivel socioeconómico y mayor número de compañeros 
sexuales, este estudio no permitió analizar estas asociaciones por la baja tasa de respuesta en los casos con relación a dichas variables.

Al año del diagnóstico se encontró que $20.99 \%$ (17) de las 81 mujeres con cáncer cervical incluidas en el estudio habían muerto. Los autores del estudio explican la alta mortalidad por el grado tan avanzado del cáncer momento en el cual se hace el diagnóstico en las mujeres atendidas en el Hospital San Vicente de Paúl y consideran que la probabilidad de muerte en mujeres con cáncer de cervix podría disminuirse si existiera un buen programa de Vigilancia Epidemiológica para esta entidad. Uribe (14) describe una mortalidad de $17.02 \%$ a los cinco años de seguimiento en una cohorte de 2.255 mujeres con cáncer de cuello uterino.

La mortalidad en Cuba según lo reportado por Alert (29) en 728 pacientes con diagnóstico de cáncer cervical fue de 34.7 por ciento a los cinco años de seguimiento. Hill (36) en revisión de estadísticas mundiales de la Organización Mundial de la Salud, Deutsch (38) y Pérez (39) en Venezuela, informan un descenso en la mortalidad por cáncer cervicouterino a partir de los programas masivos de control de cáncer. En una revisión de 278 casos de cáncer in-situ de cervix, diagnósticos en diferentes instituciones de salud en Bogotá según referencia (27), se encontró una mortalidad 0.6 por ciento, en pacientes en quienes sólo el 10 por ciento llevaba cinco años de seguimiento y el resto no había alcanzado este período después del diagnóstico.

Chisto-Pherson (30), reporta una mortalidad en 91 pacientes con cáncer de cervix de 5.49 por ciento a los cinco años de seguimiento y a los 10 años la mortalidad fue de $\mathbf{1 2 . 5}$ por ciento.

\section{RESUMEN}

Estudio de casos y controles realizado en 81 pacientes con cáncer de cuello uterino, diagnosticados en el Hospital San Vicente de Paúl de la ciudad de Medell ín y 162 controles, mujeres con informe de citología negativa o grados I ó II sin atipias procesadas en el mismo hospital. El $88.99 \%$ eran cáncer invasor, $9.88 \%$ cáncer in-situ. Como factores de riesgo se estudiaron las siguientes variables: edad, procedencia; edad a la primera relación sexual, primer embarazo la menarquia; multiparidad, consumo de anovulatorios, infecciones genitales, abortos, trastorno menstrual $y$ antecedentes familiares. De ellos sólo se analizaron las seis primeras variables en las cuales el índice de respuesta fue similar para casos y controles. Se encontró asociación estadística con las variables de procedencia, edad a primera relación sexual y el primer embarazo y multiparidad. Como estad ígrafo de contraste se usó una diferencia de proporciones y promedios y un nivel de significancia de 0.05 .

\section{CERVICAL CARCINOMA AND ITS RISK FACTOR CONTROL AND CASE STUDY}

\section{Summary}

A control and case study was made on 81 patients with cancer in the uterine neck, who were diagnosed in Hospital San Vicente de Paul in Medell ín. 162 controls were also made in that Hospital for women with negative cytology or degrees I or II without athypias. $88.99 \%$ showed invading cancer and $9.88 \%$ had cancer in situ. The following variables were analyzed as risk factors: age, origin; age in the first sexual relation, first pregnancy and menarche; multiparity, anovulatory consumption, genital infections, abortions, menstrual disorders and family background. Only the first six variables were analyzed, showing a res- 
ponse index similar to that of cases and controls. A statistical relation was found in the variables of origin, age in the first sexual relation and in the first pregnancy and multiparity. A difference in ratios and averages and a 0.05 significancy level were used as statistical graphs of contrast.

\section{Agradecimientos:}

- Grupo de Oncología Ginecológica Universidad de Antioquia.

- Doctor Jaime Uribe D. Profesor Departamento de Ginecología y Obstetricia. Facultad de Medicina, Universidad de Antioquia.

- Dra. Constanza Díaz de Calle, Jefe Departamento de Anatomía Patológica. Hospital San Vicente de Paúl.

\section{Bibliografía}

1. NAHMIAS, André J. NAIB, Zuherm, et al., "Epidemiological studies relating genital Herpetic Infection to cervical carcinoma. Cancer research. Baltimore 34 (5): 1111-1117, 1974.

2. GUZMAN, Ney y GUERRERO, Rodrigo. "Cáncer de Cervix Uterino en Cali, CoIombia", Antioquia Médica, 21: 765-786, 1971.

3. SEOTT W. JORDAN y otros. "Cáncer de Cervix en mujeres indias americanas". A Journal of the american cancer society, Philadelfia, 23 (15), 1969.

4. WRICHT N. et al. "Neoplasia and Dysplasia of the cervix uteri and contraception". The British Journal of cancer, Oxford 38 (2): 237-279, 1978.

5. NEALON, Nancy, et al. "Cervix cancer precursors in young off spring of LowIncome families". Jou rnal of the American Collage of Obstetricians and Giencologists, New York 54 (2): 135-139, 1979.
6. LEKIAN, Sibory and Wakefiel. "Incidence of cervical cancer by marital status". Journal of Epidemiology and Comunity Health, Londres, 32 (2): 108-110, 1978.

7. JAMES A, Sebastian et al. "Cancer of the cervix asexually trasmitted disease". American Journal of obstetrics and Ginecology. (St Louis) 131 (6): 620-623, 1978.

8. DAVID B. Thomas et al. "Relación del herpes simple tipo 2 y displasias escamosas en el carcinoma cervical in-situ". Journal of American cancer society, Filadelfia. 42 (6): 2716-2725, 1978.

9. TOBIN, et al. Organ culture model for the study of $\mathrm{HVH}-1 \mathrm{l}$, infections in carcinoma of the cervix". Obstetrics and Gynecology 53(5): 559-564 may, 1979.

10. ROJAS M. William. Inmunología. Ed. Col. Medellín, 1973.

11. WINDOR, Ernest et al. "A study of environmetal factors in carcinoma of the cervix". Am. Jour Obst. Gvn. 68: 10161052, 1954

12. PERITZ, Eric et al. "The Incidence of cervical cancer and duration of oral contraceptive USA". Am. You. Epi. 106 (6): 462-469, 1977.

13. SAULNY de Jorgez, Jacqueline. "Anticonceptivos Inyectables". Rev. de Obstetricia y Ginecología de Venezuela. 36 (1): 230, 1976.

14. MAtTingly F. F., Stafl A. "Riesgo del cáncer en el descendiente expuesto a dietilestibestrol". Sinopsis Obstétrico Ginecológica. XXIV (3): 248-253, 1977.

15. HERBEST, Artur L. et al. "Factors related to survival in 84 registry cases of clear cell adenocarcinoma of the vagina en cervix". Am Jour of Obst. an Gyn. 135 (7): 877-886, 1979.

16. MUÑOS y otros. "Anticuerpos contra herpes virus en pacientes con carcinoma de cuello uterino y condiloma acum ínado y controles". Acta Médica del Valle. 4: 103-106, 1975. 
I\%. GUZMAN, Ney. "Tendencias del cáncer de cervix uterino en Cali, Colombia". Antioquia Médica, 24 (3): 249-257, 1974.

18. SERVICIO SECCIONAL de Salud de Antioquia. Boletín Epidemiológico, año IV.No. 2 y 3, 1979.

19. URIBE D., Jaime. "Estudio estadístico de lesiones premalignas de cuello". Revista Colombiana de Obstetricia y Ginecología. Bogotá 29 (3): 119-138 mayojunio, 1978.

20. BOTERO Uribe, Jaime y otros. “Carcinoma de cuello uterino en el Hospital Universitario San Vicente de Paúl, Mede"l ín". Revista Colombiana de Obstetricia y Ginecología. 20 (6): 411-415, nov. dic., 1969.

21. BAQUERO, Eduardo et al. "Carcinoma In-situ del cuello uterino". Revista Colombiana de Obstetricia y Ginecología XXV (2): 95-115 marzo-abril, 1974.

22. HENAO A. Fabio y otros. "Doce años de cirugía radical por cáncer de cervix en el Hospital de San Juan de Dios de Cali". Revista Colombiana de Obstetricia y Ginecología. Bogotá 28 (4): 150-158 agosto, 1977 .

23. ERAZO, José Vicente y otros. "Variables Epidemiológicas Relacionadas con el cáncer de Cuello Uterino en pacientes con citologia III - IV - V en Popayán 1971" Revista Colombiana de Obstetricia y Gi- necología. (Bogotá) 23 (6): 553-563 nov.-dic. 1972.

24. FONNEGRA M., Alvaro y otros. "Histerectomía Radical para Carcinoma Invasivo de Cuello Uterino". Experiencia de 11 años. Revista Colombiana de Obstetricia y Ginecología. (Bogotá) 23 (2): 117125, marzo-abril, 1972.

25. RENGIFO P., Jaime. "Carcinoma de Cue Ilo Uterino". Revista Colombiana de Obs. tetricia y Ginecologia. $28(2)$ : 73-78, ju. lio, 1977.
26. BARROS P. Franco. "Carcinoma Cervical Intraepitelial". Revista Colombiana de Obstetricia y Ginecología. Bogotá 31 (1): 50-62 enero-febrero 1980.

27. VI CONGRESO NACIONAL de cirugía en Medellín. "Trabajo Cooperativo Carcinoma in-situ del Cervix". Revista Colombiana de Obstetricia y Ginecología. Bogotá 22 (2): 71-79 marzo-abril, 1971.

28. CAORSI Ch., Italo y otros. "El cáncer cervico-uterino en el sur de Chile". Boletín de la Oficina Sanitaria Panamericana. Washington 80 (1): 68-77, enero, 1976.

29. ALERT. S., José et al. "Cáncer cervicouterino: Estudio de la supervivencia en 728 pacientes". Revista Cubana de Obstetricia y Ginecología. (La Habana) 3 (4): 257-267 oct.-dic.., 1977.

30. CHISTO-PHERSON $M$, William, et. al "Microinvasive Carcinoma of the Uterine Cervix, a long tern follow-up study of eighty cases". Cancer (Philadelphia) 38 (2): 629-632, Augost, 1976.

31. RAMIREZ G., Rafael et al. "Estudio Analítico de la Biopsia Cervical Multifragmentaria". Asociación de Ginecología y Obstetricia. (México) 44 (264): 327-335 oct. 1978.

32. MARCOS, C. et al. "El Diagnóstico Citológico en el Cáncer de Utero". Revista Española de Obstetricia y Ginecología. (Barcelona) 38 (244): 172-180, marzo, 1979.

33. RUCH. M. et al. "Carcinoma Microinvasor del cuello". Sinopsis Obstétrico Ginecológica. (Buenos Aires) 23 (3): 228-232, 1976.

34. AURE Iulene, Cesar, et al. ".Carcinoma de Cuello". Boletín de la Sociedad Vene zolana de Cirugía. (Caracas-Venezuela) Volúmen $24(1-5)$ : 125-139, enerooctubre, 1975.

35. BEDOYA H. Mariano, et al. "Epidemiología del Cáncer del Cervix en el Perú". Revista Colombiana de Obstetricia y Ginecología. Vol. 23 (5): 411-420, sept oct., 1972. 
36. HILL Gerry B. “Cáncer de Utero, tendencias de la mortalidad desde 1950". Crónica de la O.M.S. (Ginebra) 30 (5); $201-$ 206.

37. KODAMA, Mitsou, et al., "Relationship betwen epidemiologic and endocrinologic aspect of cervical cancer". Journal of the National Cancer Institute. Washington, 61 (1): 35-39, jul., 1978.

38. DEUTSCH, Nolvin, et al. "The Changing
Trends of Morbidity and Mortality Trends Over a Twenty Year Period". A Journal of the American Cancer Society. Philadelphia-Toronto, 42 (5): 2439-2449, noviembre, 1978.

39. PEREZ A., Luis, et al. "Situación del Cáncer Ginecológico y de la mama y evolución de su campaña de pesquisa y $D x$. precoz quinquenio 1966-1970". Salud Pública de Caracas, (Venezuela) 47 (1): 21, 1971. 